\title{
Distributions of ischemic heart disease risk factors in patients who were admitted for angioplasty in Iran*
}

\author{
Ahmad Reza Assareh ${ }^{1}$, Maria Cheraghi ${ }^{2}$, Mohammad Nourizadeh ${ }^{1}$, Farzad Daeenejad $^{1}$, \\ Habib Haybar ${ }^{3 /}$, Mohammad Reza Kiarsi ${ }^{1}$ \\ ${ }^{1}$ Imam Khomeini Hospital, Ahvaz Jundishapur University of Medical Sciences, Ahvaz, Iran \\ ${ }^{2}$ Public Health Department, Ahvaz Jundishapur University of Medical Sciences, Ahvaz, Iran \\ ${ }^{3}$ Cardiovasclar Disease Research Center, Ahvaz Jundishapur University of Medical Sciences, Ahvaz, Iran \\ Email: "Takamoolsk@yahoo.com
}

Received 11 April 2013; revised 20 May 2013; accepted 30 May 2013

Copyright (C 2013 Ahmad Reza Assareh et al. This is an open access article distributed under the Creative Commons Attribution License, which permits unrestricted use, distribution, and reproduction in any medium, provided the original work is properly cited.

\begin{abstract}
The aim of this study was to study the prevalence of different risk factors of coronary artery disease (CAD) in Patients who were admitted for angioplasty. Methods: A cross-sectional study was conducted on 333 patients who were admitted with diagnosis as a CAD and candidates for angioplasty in Imam Khomeini hospital, Ahvaz city, Iran 2011. The prevalence of different risk factors was determined using descriptive statistics. Results: The prevalence of CAD risk factors in overall subjects were: 1) $45.3 \%$ hypertension (considering $>140 / 90 \mathrm{mmHg}$ ); 2 ) $34.5 \%$ high cholesterol (>240 mg/dl); 3) $27.6 \%$ diabetic mellitus $(>126 \mathrm{mg} / \mathrm{dL})$; 4) family history of heart disease: $20.7 \%$; 5) smokers: $19.9 \%$. Conclusion: This data have shown the prevalence of IHD (Ischemic Heart Disease) risk factors specially about hypertension in patients with IHD was high, so could require urgent decision making to national control program of noncommunicable diseases in Iran.
\end{abstract}

Keywords: Prevalence; (Ischemic Heart Disease) Risk Factors; Ahvaz; Iran

\section{INTRODUCTION}

Cardiovascular diseases are a major public health problem worldwide [1]. CAD is the most common form of cardiovascular disease with an estimated prevalence of CAD in men is $6.9 \%$, and $6 \%$ among women [2]. CAD is the major cause of morbidity and mortality and accounts for the most expensive costs in medical assistance in Iran

${ }^{*}$ Competing interests: The authors declare that they have no competing interests.

${ }^{\#}$ Corresponding author.
[3]. It is responsible for approximately $30 \%$ of all deaths worldwide each year [4]. Almost $80 \%$ of these deaths occur in low and middle income countries, and half occur in women [5].

$\mathrm{CAD}$ is a chronic process that begins during adolescence and slowly progresses throughout life. Independent risk factors include family history of premature CAD, cigarette smoking, diabetes mellitus, hypertension, dyslipidemia, a sedentary life style, advanced age, gender and obesity. The risk factors accelerate or modify a complex and chronic inflammatory process that ultimately manifests as fibrous atherosclerotic plaque [2]. The incidence of CAD is compatible with the pattern of the distribution of CAD risk factors, it occurs when the risk factors are present, so we need to know the prevalence of these risk factors, isolated or in combination, because it is through the reduction of these risk factors and using primary and secondary prevention programs that we will achieve effectiveness in any health program. The familial antecedents are un-modifiable and independent risk factors that, even though having already been studied [6], still require further investigation. Patients whose firstdegree relatives develop early CAD have higher risks of developing coronary artery disease than the general population.

Cigarette smoking may lead to double risk for coronary artery disease, $30 \%$ of which are attributed to the number of cigarettes smoked. In a study $[7,8]$ with 106,745 males in Korea, tobacco was a major and modifiable risk factor for cardiovascular disease, according to serum cholesterol levels; low cholesterol levels did not provide a protective effect in those smokers. These findings are supported by those of the prospective study of the American Cancer Society, of the Nurses' Health Study, and of some meta-analyses [9-12]. Coronary ar- 
tery disease occurs more commonly in diabetic patients than in the general population, affecting more than 55\% of patients [13].

Hypertension is a well-established risk factor for cardiovascular disease and for congestive heart failure [14]. The importance of this association was well defined in the findings of the Framingham Study and Multiple Risk Factor Intervention Trial-MRFIT [15].

The aim of this study was to investigate the prevalence of different CAD risk factors in Patient, who were admitted for angiography in Imam Khomeini Hospital, Ahvaz city.

\section{METHODS}

A cross-sectional study was conducted involving 333 patients who were admitted and diagnosed as CAD candidate for angiography in Imam Khomeini Hospital, Ahvaz city, 2011.

Inclusion criteria for angiography were patients with CAD risk factors that had high risk characteristics in non invasive test such as exercise treadmill test (ETT) or they had typical chest pain with elevated cardiac enzyme.

Patients with neurologic dysfunction or sever peripheral artery disease or sever renal function impairment were excluded from study.

This study was conducted to assess the prevalence of CAD risk factors utilizing the medical history, physical examination and laboratory tests to consider known risk factors.

We collected the data regarding risk factors for all 333 patients. After completing their informed consent, they were given an interview, physical examination and blood sample tests. Age was confirmed as per identify card. Preprotocol blood samples were obtained for FBS and total cholesterol. FBS was assayed at the enzymatic methodology by glucose oxidize Kit; total cholesterol was assayed at the enzymatic methodology.

Arterial blood pressure levels in the right arm (average of 3 measurements having the patient seated and rested), total cholesterol, lipoprotein profile and fasting glucose concentrations. The measurements were collected during the first $72 \mathrm{~h}$ of hospitalization. Individuals' past medical histories and their reports assisted us in characterizing the subjects as having hypertension, hypercholesterolemia, or diabetes. In keeping with the long-standing classification criteria used in several population-based studies, patients whose blood pressure, according to their medical records, were greater or equal to $140 / 90 \mathrm{mmHg}$ or were taking antihypertensive medication were classified as hypertensive [13].Hypercholesterolemia and hypertriglyceridemia were defined as serum total cholesterol (TC) and triglycerides (TG) levels greater than 200 and $150 \mathrm{mg} / \mathrm{dl}$, respectively, or if hypo-lipidemic treat- ment was administered. Diabetics were those with fasting blood glucose equal or greater than $126 \mathrm{mg} / \mathrm{dl}$ for two times or those who were under diabetic diet or medications.

\section{ETHICS}

The study protocol was approved by ethics committee of Ahvaz Jundishapur University of Medical Sciences. All patients provided written informed consent.

\section{STATISTICAL ANALYSIS}

Prevalence of CAD risk factors was determined using descriptive statistics, and it was used to process the outcomes in tables and graphs. All analysis was performed using SPSS [16] statistical analysis software.

\section{RESULTS}

We assess 333 CAD patients those were admitted for angiography in Imam Khomeini Hospital in Ahvaz city, out of them $59.2 \%$ was male, and $40.8 \%$ was female. Table 1 shows the basic characteristics of patients.

Figure 1 demonstrated the prevalence of CAD risk factors under the study including, hypertension, hyperlipidemia, diabetic mellitus, family history of CAD in the

Table 1. Basic characteristics of patients.

\begin{tabular}{ccc}
\hline & Number & Percent \% \\
\hline age & $59.12 \pm 13.1$ & \\
male & 197 & 59.2 \\
smoker & 66 & 19.8 \\
HTN & 151 & 45.3 \\
HLP & 115 & $34 / 5$ \\
DM & 92 & 27.6 \\
FH & 69 & $20 / 7$
\end{tabular}

HTN: Hypertension, HLP: Hyperlipidemia, DM: Diabetes mellitus, FH: Family history.

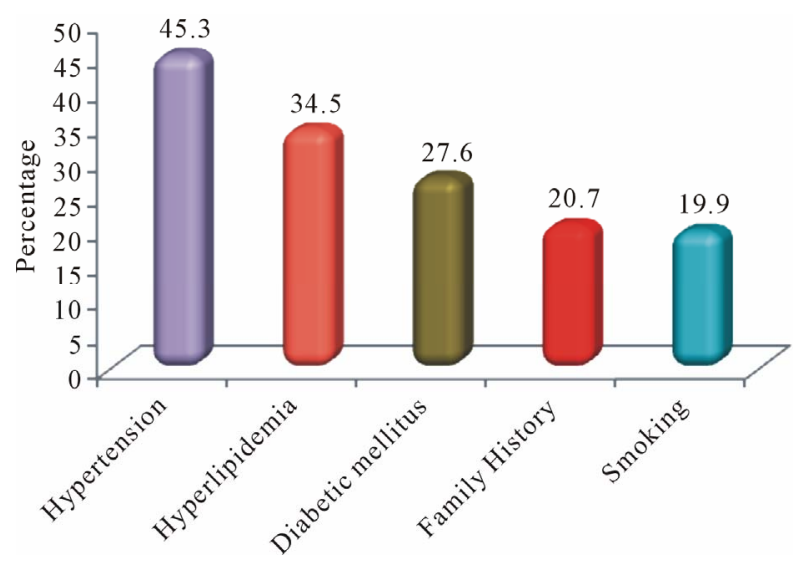

Figure 1. The prevalence of CAD risk factors in patients. 
first degree relative and cigarette smoking (active smoker). This data has shown that prevalence of hypertension (45.3\%) was the highest risk factors and cigarette smoking with $19.9 \%$ was lowest risk factors in CAD Patients.

Our data has shown prevalence of hypertension, hyperlipidemia, diabetes, family history of CAD in the first degree relative and cigarette smoking (active smoker) in males as well as in female (Figure 1).

These data had demonstrated that prevalence of hyperlipidemia and diabetic mellitus were in female more than male ( $p<0.05$ ), and prevalence of family history of $\mathrm{CAD}$ in the first degree relative was almost same $(\mathrm{p}>$ 0.05), but prevalence of cigarette smoking in male was 3 fold more than female p $<0.05$ (Figure 2 and Table 2).

\section{DISCUSSION}

CAD is the epidemic of current time and set to remain the single most important disease in the world in the terms of mortality, morbidity, disability and economic loss till 2020 [17]. This chronic disease has an enormous impact on quality of life. There are risk factors accelerate or modify a complex and chronic inflammatory process that ultimately manifest as fibrous atherosclerotic plaque [2], the clinical coronary events follow plaque rupturing. The presence and the number of CAD risk factors predict the future cardiovascular events in individuals with such factors (Hypertension, hyperlipideamia, diabetic mellitus

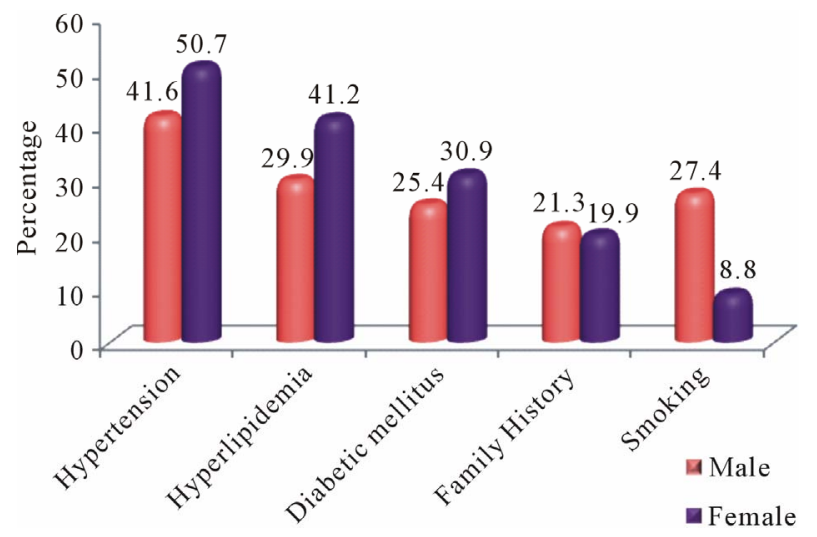

Figure 2. The prevalence of risk factors in CAD patients in male and female.

Table 2. Comparison the risk factors between male and female.

\begin{tabular}{cccc}
\hline & male & female & p value \\
\hline smoker & $27.4 \%$ & $8.8 \%$ & $<0.01$ \\
HTN & $41.6 \%$ & $50.7 \%$ & 0.051 \\
HLP & $29.9 \%$ & $41.2 \%$ & $<0.01$ \\
DM & $30.9 \%$ & $25.4 \%$ & 0.03 \\
FH & $21.3 \%$ & $19.9 \%$ & 0.5 \\
\hline
\end{tabular}

and smoking).

The main finding of this study was the high prevalence of risk factors for CAD among men and women in our population, these events might be, because of cultural and behavioural differences and different dietary habits.

There is strong evidence that in developing countries such as Iran, there has been a recent rapid increase in the prevalence of HTN. A systemic review of 29 reports with a total sample size of 93,661 subjects and the results of a very large national survey reporting the prevalence of HTN in 27 provinces in Iran estimated the overall prevalence of HTN in aged 30 - 55 years and age more than 55-year-old to be $23 \%$ and $50 \%$, respectively [16]. In the present study, the prevalence of hypertension in patients undergoing coronary angiography was more than other risk factors, this finding shows more than previous study which had done in Iran [3,18-21] and Brazil [22].

Numerous epidemiological studies have shown that the presence of hypertension increases the risk of CAD, not only in, at risk populations; but also in the general population [23].

Previous studies in Iran [24], had shown the prevalence of $60.9 \%$ of hypertension in patients undergoing coronary angiography indicates its important effect in developing CAD. Accumulation of other coronary risk factors in hypertensive patients was also shown, as diabetes mellitus and hyperlipidemia were more frequent in hypertensive patients than in normotensive persons.

There is a high frequency of dyslipidemia in Iran [18, 25]. In this study, we also observed a higher prevalence of hyperlipideamia in the patients which has confirm with a study in 2009 had shown that low serum HDL-C concentration was one of the strongest factors independently associated with CAD in the Iranian population [26]. Several reports have indicated that dyslipidemia is significantly more common in Iranian patients [26-29].

The prevalence of type 2 diabetes mellitus (DM) ranges from $1.2 \%$ to $14.6 \%$ in Asia, $4.6 \%$ to $40 \%$ in the Middle East, and $1.3 \%$ to $14.5 \%$ in Iran [26-28]. Diabetes mellitus is highly prevalent among the members of the Iranian population with established CAD [26,28,29]. Our study shows the prevalence of diabetes mellitus was 27.6\% in overall patients in Imam Khomeini Hospital in Ahvaz city. It was more common in female $(25.4 \%$ in male and $30.9 \%$ in female). This data was conformed to previous studies in Shiraz [19], Isfahan [20] and Tehran [21]. A study indicated that there is also a high prevalence of CAD among Iranian type 2 diabetic patients [30]. A study of 10,622 consecutive patients undergoing elective coronary artery bypass grafts (CABG) between 2001 and 2005 had shown that more than one third of these patients had DM [31]. Esteghamati reported that 30\% of the patients with acute coronary syndrome in Iran have 
DM [31].

During the last several years in Iran, there have beenseveral widespread studies conducted on smoking. The National Health and Disease Survey is the most extensive of these, and reported a decrease in smoking from $14.6 \%$ in 1991 to $11.7 \%$ in 1999 [32,33]. Our result indicated that the prevalence of smoking was $19.9 \%$ in overall population; this data showed the prevalence of smoking was more common in male (27.4\%) than female (8.8\%), our data on prevalence of smoking was reported less than Shiraz [19], Isfahan [20] and Tehran [21] studies.

\section{CONCLUSIONS}

We can mention that this study reflects the reality of the prevalence of the risk factors for coronary artery disease in Ahvaz city. Our study has a clinical plan based on well-documented epidemiological studies, and it stresses the actual value of the risk factors assessed for coronaryartery disease.

With the increase in mean survival of the population, chronic and degenerative diseases will be more and more present. The best treatment for the major chronic and degenerative disease, ischemic heart disease, is prevention, which is achieved by fighting the risk factors. The best way to assess our management of the risk factors for coronary artery disease is through health programs comparing the results based on actual percentages of prevalence.

\section{REFERENCES}

[1] Gus, I. and Zielinsky, P. (1999) As cardiopatias no Brasil. In: Ferreira, C. and Póvoa, R., Eds., Cardiologiapara $O$ ClínicoGeral, Atheneu, Rio de Janeiro, 131-143.

[2] Thom, T., Haase, N., Rosamond, W., Howard, V.J. and American Heart Association. (2006) Stroke statistics heart disease and update. Circulation, 113, e85-e151.

[3] Hatmi, Z.N., Tahvildari, S., GafarzadehMotlag, A. and SabouriKashani, A. (2007) Prevalence of coronary artery disease risk factors in Iran: Apopulation based survey. BMC Cardiovascular Disorders, 7, 32. doi:10.1186/1471-2261-7-32

[4] World Health Organization. (2002) The world health report 2002: Reducing risks, promoting healthy life. WHO, Geneva, 27 October 2002.

[5] Naghavi, M. and Jafari, N. (2007) Death profile in Iran, 2005. Iranian Ministry of Health, Tehran.

[6] Shimoda, M., Sugayama, S., Kim, C. and Ebaid, M. (1996) Orientação Familiar preventiva: Aspectosgenéticos das doençascardiovasculares e perspectivasfuturas. Revista da Sociedade de Cardiologia do Estado de São Paulo, 6, 623-627.

[7] Ockene, I.S. and Miller, N.H. (1997) Cigarette smoking, cardiovascular disease, and stroke. A statement for healthcare professionals from the American Heart Association. Circulation, 96, 3243-3247. doi:10.1161/01.CIR.96.9.3243

[8] Jee, S.H., Suh, I., Kim, I.S. and Appel, L.J. (1999) Smoking and atherosclerotic cardiovascular disease in men with low levels of serum cholesterol: The Korea medical insurance corporation study. The Journal of the American Medical Association, 282, 2149-2155. doi:10.1001/jama.282.22.2149

[9] Steenland, K., Thun, M., Lally, C., et al. (1996) Environmental tobacco smoke and coronary heart disease in the American Cancer Society CPS-II cohort. Circulation, 94, 622-628. doi:10.1161/01.CIR.94.4.622

[10] Kawachi, I., Colditz, G.A., Speizer, F.E., et al. (1997) A prospective study of passive smoking and coronary heart disease. Circulation, 95, 2374-2379. doi:10.1161/01.CIR.95.10.2374

[11] Law, M.R., Morris, J.K. and Wlad, N.J. (1997) Environmental tobacco exposure and ischaemic heart disease: An evaluation of the evidence. British Medical Journal, 315, 973-980. doi:10.1136/bmj.315.7114.973

[12] He, J., Vupputri, S., Allen, K., et al. (1999) Passive smoking and the risk of coronary heart diseasea meta-analisys of epidemiologic studies. The New England Journal of Medicine, 340, 920-926. doi:10.1056/NEJM199903253401204

[13] Fein, F. and Scheuer, J. (1990) Heart disease in diabetes mellitus: Theory and practice. In: Rifkin, H. and Port, D. Eds., Diabetes Mellitus, Elsever, New York, 812-823.

[14] Aram, V. Chobanian, M.D. and Bakris, G.L. (2003) The 7th Report of the Joint National Committee on Prevention, Detection, Evaluation, and Treatment of High Blood Pressure: The JNC 7 Report. JAMA, 289, 2560-2571.

[15] Wilking, S.V.B., Belanger, A.J., Kannel, W.B., D’Agostinho, R.B. and Steel, K. (1988) Determinants of isolated systolic hypertension. The Journal of the American Medical Association, 260, 3451-3455. doi:10.1001/jama.1988.03410230069030

[16] Haghdoost, A.A. Sadeghirad, B. and Rezazadehkermani, M. (2008) Epidemiology and heterogeneity of hypertension in Iran: A systematic review. Archives of Iranian Medicine, 11, 444-452.

[17] Gazino, J.M. (2005) Global burden of cardio vascular disease. In: Braunwald, E., Ed., Heart disease: A textbook of cardiovascular medicine, USA 7th Edition, WB Saunders Company, Philadelphia, 7.

[18] Sarraf-Zadegan, N., Amboshtam, M. and Rafiei, M. (1999) Risk factors for coronary artery disease in Isfahan, Iran. European Journal of Public Health, 9, 20-26. doi:10.1093/eurpub/9.1.20

[19] Azizi, F., Rahmani, M., Emami, H., Mirmiran, P., Hajipour, R., Madjid, M., et al. (2002) Cardiovascular risk factors in an Iranian urban population: Tehran lipid and glucose study (phase 1). SOZ Preventive Medicine, 47, 408-426. doi:10.1007/s000380200008

[20] Baguet, J.P., Barone-Rochette, G. and Mallion, J.M. (2006) European society of hypertension scientific news- 
letter: Hypertension and coronary heart disease. Journal of Hypertension, 24, 2323-2325. doi:10.1097/01.hjh.0000249719.05006.58

[21] Akbarzadeh, F., Pourafkari, L., HashemiJazi, S.M., Hesami, L. and Habibi, V. (2010) Prevalence and severity of coronary of artery disease among hypertensive and normotensive patients. ARYA Atherosclerosis Journal, 5, 186-190.

[22] Gus, I., Fischmann, A. and Medina, C. (2002) Prevalence of risk factors for coronary artery disease in the brazilian state of rio grande do sul, Arquivos Brasileiros de Cardiologia, 78, 484-90. doi:10.1590/S0066-782X2002000500005

[23] Thelle, D.S., Shaper, A.G. and Whitehead, T.P. (1983) Blood lipids in middle-aged British men. British Heart Journal, 49, 5-13.

[24] Sharifi, F., Mousavinasab, S.N., Soruri, R., Saeini, M. and Dinmohammadi, M. (2008) High prevalence of low high-density lipoprotein cholesterol concentrations and other dyslipidemic phenotypes in an Iranian population. Metabolic Syndrome and Related Disorders, 6, 187-95. doi:10.1089/met.2008.0007

[25] Ebrahimi, M., Kazemi-Bajestani, S.M., Ghayour-Mobarhan, M., Moohebati, M., Paydar, R., Azimi-Nezhad, M., et al. (2009) Metabolic syndrome may not be a good predictor of coronary artery disease in the Iranian population: Population-specific definitions are required. The Scientific World Journal, 9, 86-96. doi:10.1100/tsw.2009.17

[26] Kazemi-Bajestani, S.M., Ghayour-Mobarhan, M., Ebrahimi, M., Moohebati, M., Esmaeili, H.A., Parizadeh, M.R., et al. (2007) Serum copper and zinc concentrations are lower in Iranian patients with angiographically defined coronary artery disease than in subjects with a normal angiogram. Journal of Trace Elements in Medicine and Biology, 21, 22-28. doi:10.1016/j.jtemb.2006.11.005

[27] Ghayour-Mobarhan, M., Sahebkar, A., Parizadeh, S.M., Moohebati, M., Tavallaie, S., Rezakazemi-Bajestani, S.M., et al. (2008) Antibody titres to heat shock protein 27 are elevated in patients with acute coronary syndrome. International Journal of Experimental Pathology, 89, 209-215. doi:10.1111/j.1365-2613.2008.00586.x

[28] Parizadeh, S.M., Moohebati, M., Ghafoori, F., GhayourMobarhan, M., Kazemi-Bajestani, S.M., Tavallaie, S., et al. (2009) Serum selenium and glutathione peroxidase concentrations in Iranian patients with angiography-defined coronary artery disease. Angiology, 60, 186-191.

[29] Janghorbani, M., Amini, M. and Tavassoli, A. (2006) Coronary heart disease in type 2 diabetes mellitus in Isfahan, Iran: Prevalence and risk factors. Acta Cardiologica, 61, 13-20. doi:10.2143/AC.61.1.2005135

[30] Karimi, A., Marzban, M., Movahedi, N., Salehiomran, A., Sadeghian, S. and Goodarzynejad, H. (2009) Traditional cardiac risk factors profile in Iranian patients undergoing coronary artery bypass surgery. Acta Cardiologica, 64, 371-377.doi:10.2143/AC.64.3.2038024

[31] Mohammad, K., Zali, M., Masjedi, M. and Majdzadeh, S. (1998) Cigarette smoking in Iran based on a national health survey. Medical Journal of the Islamic Republic of Iran, 1, 33-37.

[32] Mohammad, K., Noorbala, A., Majdzadeh, S. and Karimloo, M. (2001) Trend of smoking prevalence in Iran from 1991 through 1999 based on two national health surveys. Hakim Research Journal, 4, 290-297.

[33] Amani, R., Nourizadeh, M., et al. (2010) Nutritional related cardiovascular risk factors in patients with coronary artery disease in Iran: A case-control study. Nutrition Journal, 9, 70. 